\title{
Ileal bile acid transporter inhibitor improves hepatic steatosis by changing the gut microbiota dysbiosis in NAFLD model mice
}

\section{Masahiro Matsui}

2nd department of internal medicine, Osaka Medical College

\section{Shinya Fukunishi}

2nd department of internal medicine, Osaka Medical College

\section{Takashi Nakano}

Department of Microbiology and infection control, Osaka Medical College

\section{Takaaki Ueno}

Department of Oral surgery, Osaka Medical College

\section{Kazuhide Higuchi}

2nd department of internal medicine, Osaka Medical College

Akira Asai ( $\nabla$ in2108@osaka-med.ac.jp )

Osaka Medical College https://orcid.org/0000-0002-2915-7875

\section{Research}

Keywords: non-alcoholic fatty liver disease, ileal bile acid transporter inhibitor, gut microbiome

Posted Date: April 21st, 2020

DOI: https://doi.org/10.21203/rs.3.rs-21966/v1

License: (c) (i) This work is licensed under a Creative Commons Attribution 4.0 International License. Read Full License 


\section{Abstract}

\section{Background \& Aim:}

Non-alcoholic fatty liver disease (NAFLD) which is characterized by excessive fat deposition in the liver that is not attributable to consumption of alcohol, highly prevalent in all countries. However, therapeutic agents approved for the treatment of NAFLD are lacking. An ileal bile acid transporter inhibitor (IBATi) which represents a new mode of treatment of chronic idiopathic constipation, leads to increased delivery of bile acids to the colon. We investigated the effect of IBATi for NAFLD through modification of the gut microbiota in mice.

\section{Results}

When HFD mice were treated with IBATi, their body weight gain and serum LDL levels were significantly suppressed and NAFLD activity score was significantly decreased. Treatment with IBATi ameliorated the increase of ileal Fgf15 mRNA and the decrease hepatic Cyp7a 1 mRNA in HFD mice. The microbial compositions of the feces from these mice were analyzed using 16S rRNA sequencing. The decrease of a-diversity in gut microbiota induced by HFD was recovered by the IBATi treatment. To establish a causeeffect of the improvement efficacy of IBATi for NAFLD, we recolonized antibiotic solution-treated mice reared in SPF conditions by fecal microbiome transplantation (FMT) using stool from the HFD or HFD + IBATi mice. From FMT, the gut microbiota from HFD + IBATi mice prevented hepatic steatosis caused by HFD.

\section{Conclusions}

IBATi improves hepatic steatosis by changing the gut microbiota dysbiosis in NAFLD model mice.

\section{Background}

During the past century, dramatic modifications in lifestyle have radically changed the health priorities in most areas of the world, owing to a growing incidence of non-communicable diseases. The new epidemic of chronic liver disease is related to the burden of non -alcoholic fatty liver disease (NAFLD) [1, 2]. NAFLD is characterized by excessive fat deposition in the liver that is not attributable to consumption of alcohol; the most common risk factors include obesity, insulin resistance, and the features of metabolic syndrome. Community surveys, based on ultrasonography or proton NMR spectroscopy, have assessed the prevalence of NAFLD across various geographical regions. NAFLD is highly prevalent in all continents, but the highest rates are reported from South America (31\%) and the Middle East (32\%), followed by Asia (27\%), the USA (24\%) and Europe (23\%), whereas NAFLD is less common in Africa (14\%) [1]. Most liver-related outcomes occur once cirrhosis has developed, with the exception of hepatocellular 
carcinoma (HCC) that can develop even in the absence of cirrhosis, and NASH is rapidly increasing as an etiology of liver cirrhosis [3].

The first-line treatment for NAFLD is lifestyle modification to achieve weight reduction, particularly through diet and exercise. However, weight reduction is very difficult to achieve and maintain and therapeutic agents approved for the treatment of NAFLD are lacking[4]. The ileal bile acid transporter (IBAT), also called apical sodium-dependent bile acid transporter (ASBT), is a key element in the enterohepatic circulation of bile acids (BAs) and treatment with a locally-acting IBAT inhibitor (IBATi) leads to increased delivery of BAs to the colon and represents a new mode of treatment of chronic idiopathic constipation[5]. It has been reported recently that the inhibition of ileal bile acid uptake protects against non-alcoholic fatty liver disease in mice [6]. However, the effect of IBATi against NAFLD through the gut microbiota is not unclear.

There is an anatomical link between the intestine and liver via the hepatic portal system. Based on this connection between the intestine and liver, also termed the gut-liver axis, gut microbiota and their metabolic products may influence liver pathology and this axis plays a pivotal role in the pathogenesis of NAFLD $[7,8]$. The human gut microbiota contains over 100 fold more genes than its host and has been also suggested to be an important environmental factor involved in the pathogenesis of NAFLD[9]. However, there is no report of how IBATi affects the gut microbiota in NAFLD.

The composition of the gut microbiota is partially modulated by extracellular metabolites that are derived from the host and modified by microbes. Of these, bile acids (BAs) constitute a highly abundant pool of host-derived and microbial-modified metabolites that are major regulators of the gut microbiota. BAs are synthesized from cholesterol in the liver, are secreted into bile as the major solute, and function to facilitate lipid absorption in the intestine. About $95 \%$ of intestinal BAs are reabsorbed in the ileum by the IBAT and conveyed through the portal vein to the liver, where they are taken up by hepatocytes to be resecreted into the bile. BAs are antibacterial and constitute strong selective forces on the intestinal microbiota. Even within a single bacterial species, there can be differential sensitivity to specific BAs. Dietary fat content can regulate the time and amount of secreted bile and thus shape the microbiota [10]. In this study, we investigated the effect of IBATi for NAFLD through modification of the gut microbiota.

\section{Materials And Methods}

\section{Mice}

All animal experiments were carried out in compliance with ICLAS guideline and Japanese regulations. The local institutional animal ethics board of Osaka Medical college approved all mouse experiments (approval number: 2019 - 138). Five week-old male C57BL/ 6 mice were purchased from Japan SLC, Inc. All mouse experiments were performed at the animal facility in Osaka Medical college, where the animals were housed in specific pathogen-free conditions. The mice were divided into four groups of 6 and fed either a control diet (CE-2; CLEA Japan) or an HFD (HFD32; CLEA Japan), for 12 weeks. For the IBATi and 
HFD + IBATi groups, the mice were treated with Elobixibat (EA Pharma Co. Ltd, Japan) $(2.5 \mu \mathrm{mol} / \mathrm{kg}$ : corresponding to the clinically optimal dose for patients) by oral gavage in $0.2 \mathrm{ml}$ of PBS daily, using animal feeding needles.

For some experiments, 6 week-old C57BL/ 6 mice were fed an HFD for 12 weeks and treated with oral IBATi from 6 to 12 weeks (IBATi-Tx group). For the control group, the equivalent mice were subjected to the same oral dose of PBS.

\section{Metagenomic analysis of mouse feces.}

Fresh feces were collected, placed into tubes, and kept at $-80^{\circ} \mathrm{C}$ until further use. Bacterial genomic DNA was extracted from fecal samples, as previously described [11]. Briefly, whole bacterial DNA was extracted from the feces using a PureLinkTM Micobiome DNA Purification Kit (ThermoFisher Scientific K. K. Tokyo, Japan).

\section{Fecal Microbiota Transplantation}

Elobixibat has only $2 \%$ bioavailability and is excreted from the body 48 hours after oral administration. When absorbed, elobixibat is protein bound $(>99 \%)$ in plasma, with a half-life of less than 4 hours [12] [13]. For fecal microbiota transplantation (FMT), feces were collected from the 12 week-HFD group or HFD + IBATi group of mice, 48 hours after the last oral administration of elobixibat, and stored at $-80^{\circ} \mathrm{C}$. Six week-old male C57/B6 mice were fed an HFD for 8 weeks. At 9 weeks of age, these mice were treated with an antibiotic solution (ATB) containing ampicillin ( $1 \mathrm{mg} / \mathrm{ml})$, streptomycin ( $5 \mathrm{mg} / \mathrm{ml}$ ) and colistin $(1 \mathrm{mg} / \mathrm{ml})$, added to their sterile drinking water. Solutions and bottles were changed 3 times per week. To investigate the efficacy of FMT, these 10 week-old "avatar mice" received ATB before FMT, and ATB treatment was discontinued $48 \mathrm{~h}$ before the first FMT[14]. FMT was performed by thawing fecal materials and $500 \mu \mathrm{L}$ of fecal suspension ( $50 \mathrm{mg} /$ mouse) was then transferred by oral gavage, using animal needles, into each ATB pre-treated recipient three times every two days, for one week.

\section{Library Preparation And Dna Sequencing}

Preparation of the library for DNA sequencing was conducted as previously described [15] using ionproton technology (ThermoFisher Scientific K. K. Tokyo, Japan). An lon 16 ${ }^{\mathrm{Tm}}$ Metagenomics Kit was used to amplify hypervariable regions from the polybacterial samples, according to the manufacturer's protocol. Library preparation was performed from $50 \mathrm{ng}$ pooled amplicons for each sample using an lon Plus Fragment Library Kit. The StepOnePlus ${ }^{\mathrm{TM}}$ Real-time PCR system was used to perform the quantitative polymerase chain reaction amplification.

\section{Sequence Data Analysis}


Sequence data analysis was performed using QIIME v1.9.0, as previously described [15]. Statistical differences $(p<0.05)$ in the relative abundance of bacterial phyla and genera between groups were evaluated using Student's paired $t$ test. The Shannon phylogenetic diversity indices were calculated using the R "phyloseq" package and statistically analyzed using a Kruskal-Wallis test followed by the SteelDwass post hoc test. The $\beta$-diversity was estimated using the Bray Curtis distances between the samples and visualized by principal coordinate analysis ( $\mathrm{PCOA}$ ); it was statistically examined using permutational analysis of variance (PERMANOVA). The final figures were generated using QIIME v1.9.0.

\section{Histological Analysis}

The liver tissue samples were fixed in $20 \%$ formalin and embedded in paraffin. Sections ( $4 \mu \mathrm{m}$ thick) were cut and stained with hematoxylin-eosin (HE). Histological features and the fat area in liver tissue sections were determined using a BZ-X 710 fluorescence microscope (Keyence, Osaka, japan). Blinded histologic assessment of slides was performed by an experimented hepatopathologist. Hepatic steatosis was evaluated by NAFLD activity score (NAS) [16] and hepatic fibrosis was evaluated by fibrosis stage[17].

\section{Serum Biochemical Analysis}

Serum was obtained from each mouse. Concentrations of aspartate amino-transferase (AST), alanine amino-transferase (ALT), Total Bile Acid (TBA), total cholesterol (Tcho), low-density lipoprotein (LDL) and triacyl glyceride (TG) in serum were measured by ELISA. ELISA kits were purchased from Elabscience (TX., U.S.A.) and MyBiosource, Inc. (CA., U.S.A.).

\section{Real-time Pcr}

Liver and terminal ileum samples were obtained from each group of mice. Hepatic mRNA expression (cholesterol 7-a-monoxygenase (Cyp7a1), cholesterol 8-a-monoxygenase (Cyp8a1) and farnesoid X receptor $(F X r)$ ) and ileal mRNA expression (Fxr and fibroblast growth factor 15 (Fgf15)) were measured by real-time PCR using a RNeasy kit (Qiagen K. K, Tokyo, Japan) and CYBR Green real-time PCR master mix (ThermoFisher Scientific K. K. Tokyo, Japan).

\section{Statistical analysis}

All data are presented as mean SEM. Body weight gain curves were analyzed with an ANOVA, followed by Tukey's multiple comparison tests. The Pearson's rank correlation coefficient was used to analyze the correlation of gut microbiota. These statistical analyses were performed using statistical software (JMP institute, Inc., Cary, NC). All results were considered statistically significant at $p<0.05$

\section{Results}




\section{IBATi inhibited of body weight increases and hepatic steatosis in HFD mice}

Six-week-old mice were fed for 18 weeks with either the control or HFD diet. Body weight and food consumption were monitored weekly throughout the experiments. During the experiment, the average food intake of mice in the HFD groups did not differ from the normal and HFD with IBATi treatment groups. As shown in Fig. 1A and B, body weights were significantly higher in the HFD group than the normal group after 3 weeks of treatment (i.e. at 9 weeks of age). This difference was maintained through to the end of the study. When HFD mice were treated with IBATi, their body weight gain was significantly suppressed during the 6-24 weeks period. The liver weight/body weight ratios were significantly higher in the HFD group than the normal group, and this increase was inhibited by treatment with IBATi (Fig. 1C). Livers were obtained from each group of 18 week-old mice and liver sections were stained with Hematoxylin and Eosin. Although the liver sections of the normal and IBATi groups were free of lipid droplets, increased accumulation of lipid droplets was observed in the HFD group, leading to hepatic steatosis, this increase being suppressed in the HFD + IBATi group (Fig. 1D and 1E). The hepatic crownlike structure that is one of the unique histological features of NAFLD was found only in the liver sections of the HFD group and not the other groups (Fig. 1F). Blinded histologic assessment of slides by an experienced hepatopathologist revealed that the NAFLD activity score (NAS) increased significantly from $0.33 \pm 0.58$ in the normal group of mice to $7.67 \pm 0.58$ in the HFD group and this score was lower in the HFD + IBATi group $(1.67 \pm 1.55)$ at 18 week of treatment (Fig. 1G). However, in this study, fibrosis was not seen in the liver sections of any group of mice at the end of the experiments (Fig. 1H). From these results, IBATi prevented the body and liver weight gain and improved hepatic steatosis in HFD mice.

Sera obtained from each group of 18 week-old mice were assayed using ELISA. In the HFD group of mice, serum AST and ALT concentrations were higher than the normal and IBATi groups of mice. When HFD mice were treated with IBATi, these increases were not prevented. Biochemical lipid measurements confirmed high levels of Tcho, LDL and TG in the sera of the HFD group, compared to the normal and IBATi groups. However, the serum levels of Tcho and TG in the HFD + IBATi group did not differ from the HFD group. The serum LDL levels were only significantly lower in the HFD + IBATi group. Serum levels of TBA in the HFD group did not differ from the HFD + IBATi group (Fig. 2A). Previous reports have revealed that hepatic Cyp7a1 mRNA expression, which promotes hepatic steatosis and serum LDL elevation, is reduced, and Fgf15 expression in the terminal ileum is increased, in NAFLD model mice[6] [18]. We analyzed the hepatic and ileal mRNA expression of each group of mice. Although, Cyp7a1 expression in the livers of the HFD group was decreased, treatment with IBATi increased its expression up to 3-fold in the livers of the HFD mice. Cyp8a 1 and FxrmRNA expression in the livers did not differ among the groups of mice. In the terminal ileum, Fgf15 mRNA expression was elevated in the HFD group and this elevation was reduced when the mice were treated with IBATi (Fig. 2B and 2C). These results indicate ileal Fgf15 mRNA expression was increased, and hepatic Cyp7a1 mRNA expression was suppressed in the HFD group of mice. Treatment with IBATi ameliorated the increase of ileal Fgf15 mRNA in HFD mice. 
Furthermore, the elevation of hepatic Cyp7a1 mRNA improved the hepatic steatosis and elevation of serum LDL.

\section{Effects Of Ibati On Hfd-induced Gut Microbiota Dysbiosis}

Given that the intestinal bile acid composition was likely to have altered the intestinal bacterial population, whole shotgun genome metagenomic sequencing was used to characterize the effect of IBATi on the gut microbiota dysbiosis induced by HFD. In total, 166 OTUs were detected from lon reporter high quality sequence reads. Initially, we compared a-diversity among the normal, HFD, IBATi and HFD + IBATi groups, using different indices for the observed species and the Shannon index. The Shannon index was significantly lower in the HFD group than the normal and IBATi groups and this decrease having been prevented by IBATi treatment (Fig. 3B).

Subsequently, the overall structures of the microbiota of the normal, HFD and HFD + IBATi groups were calculated using $\beta$-diversity induces for unweighted Bray Curtis distances. PCoA revealed that there were significant structural differences of the microbiota of the normal, HFD and HFD + IBATi groups in terms of the unweighted Bray Curtis distances (PERMANOVA, $p=0.001$ ) (Fig. 3C). Further analysis reveals this to be encoded by the genomes of the bacteria, an HFD was associated with a higher proportion of Streptococcaceae (Fig. 3A and 3D). From these results, the diversity of gut microbiota induced by HFD in mice was recovered, and the dysbiosis of the microbiota, induced in the mice by an HFD, were changed by the IBATi treatment.

\section{Effect Of Fmt On Nafld In Mice}

To establish a cause-effect of the improvement efficacy of IBATi for NAFLD, we recolonized ATB-treated mice reared in SPF conditions by FMT using stool from the HFD or HFD + IBATi groups of mice. Such "avatar mice" were prepared by oral gavage with feces harvested from the HFD or HFD + IBATi groups of 18 week-old mice. After FMT, both groups of C57BL/ 6 avatar mice were fed an HFD diet for 4 weeks. The body weights of both groups of mice increased in the same way and the liver weight / body weight ratios of the mice from each group did not differ at end of the experiment (Fig. 4A, 4B and 4C). Fatty changes were observed in the macroscopic photographs of the livers of avatar mice who received FMT from HFD mice. This change was not detected in the livers of avatar mice who received FMT from HFD-IBATi mice (Fig. 4D). In the histological liver sections, lipid droplets were observed in avatar mice with FMT from HFD mice, but not in avatar mice with FMT from HFD-IBATi mice (Fig. 4E). The NAS of the livers from avatar mice with FMT from HFD mice $(5.67 \pm 2.08)$ was higher than that from the equivalent mice with FMT from HFD-IBATi mice $(2.67 \pm 0.58)$ (Fig. 4F). As in Fig. $1 \mathrm{H}$, fibrosis was not seen in the liver sections of either group of mice (Fig. 4G). When comparing gut microbiota in both groups of mice, the a-diversity of avatar mice with FMT from HFD mice was reduced as same level as HFD mice, but this diversity of avatar mice with FMT from HFD-IBATi mice was kept even if these mice fed HFD. And there was a significantly different in $\beta$-diversity between the two groups (PERMANOVA, $p=0.007$ ) (Supplemental 
Fig. 1). From these results, the gut microbiota from HFD + IBATi mice prevented hepatic steatosis caused by an HFD.

\section{Therapeutic Effect Of Ibati Against Nafld In Hfd Mice}

Next, we tested the therapeutic effect of IBATi in HFD mice. Six week-old mice were fed an HFD diet for 12 weeks and, from 6 to 12 weeks, the mice received IBATi treatment (IBATi-Tx group). As a control group in this experiment, equivalent mice were injected with the same dose of PBS. The increase in body weight in the control group of mice was not seen in the IBATi-Tx group, their weights remaining stable with IBATi treatment (Fig. 5A and 5B). Liver weight / body weight ratios in the control group were increased but those in the IBATi-Tx group decreased after the IBATi treatment (Fig. 5C). Fatty changes seen in the macroscopic photographs of the livers and lipid droplets of the liver sections from the control group were prevented in the IBATi-Tx group (Fig. 5D and 5E). The NAS of the livers from the IBATi-Tx group (1.66 \pm 0.57) was improved and fibrosis was not detected in either group (Fig. 5F and 5G). Lower serum LDL levels were seen in the IBATi-Tx group. Other biological parameters in the sera did not differ between the groups (Fig. 6). Thus, the therapeutic effect of IBATi against hepatic steatosis was reproduced in HFD diet mice.

\section{Discussion}

BAs are synthesized in hepatocytes via cytochrome P450 (CYP)-mediated oxidation of cholesterol to the primary bile acid through "classical" and "alternative" pathways, in which CYP7A1 is the major enzyme, and secreted into the small intestine, where they facilitate the absorption of dietary lipids. The majority of BAs are reabsorbed from the intestine, returned to the liver via the portal venous circulation, and resecreted into the bile. The enterohepatic circulation of BAs is an extremely efficient process; less than $10 \%$ of intestinal BAs escape reabsorption and are eliminated in the feces[19]. IBAT is essential for efficient intestinal absorption of BAs and the mechanisms of alternative absorption of BAs are unable to compensate for loss of IBAT function[20]. In enterocytes in the ileum, activation of FXR by BAs reabsorbed by IBAT releases FGF15 (the mouse ortholog of human FGF19) into the portal circulation. FGF15 binds its receptor fibroblast growth factor receptor 4 (FGFR4), and inhibits CYP7A1, thus repressing bile acid synthesis in hepatocytes[7]. Here, we identified an important role of IBATi as regulator of hepatic lipid metabolism through the gut microbiota, in addition to its role in bile acid metabolism. It has been reported that the gut microbiota directly inhibits IBAT-dependent intestinal bile acid reabsorption[21]. Especially, enteropathogenic Escherichia coli (EPEC) directly inhibit IBAT and EPECinduced inhibition of IBATi is dependent on intact bacterial effector molecules, via type three secretion, into host cells[22]. However, EPEC was not detected in the feces of the HFD group of mice in this study. This mechanism by which gut microbiota regulate ASBT remains largely unknown and there may be other bacteria with similar capabilities. This area of investigation, which is in its infancy, may merit more research. 
In the ileum, IBAT mediates most of the uptake of BAs from the gut lumen across the apical brush border membranes of the enterocytes, whereas the organic solute transporter $\alpha / \beta($ OST $\alpha / \beta)$ plays a central role in mediating intestinal basolateral bile acid export. In order to inhibit bile acid reabsorption, either ASBT or OST may be controlled. Both Asbt-null and Osta-null mice exhibit impaired ileal bile acid absorption, decreased return of BAs to the liver and a decreased bile acid pool size [23, 24]. However, hepatic Cyp7a1 mRNA expression and bile acid synthesis are paradoxically suppressed in Osta-null mice, secondary to elevated ileal FGF15 production [25]. Blocking intestinal absorption of BAs at the enterocyte apical brush border membrane by IBATi suppresses ileal Fgf15 mRNA expression, induces hepatic bile acid synthesis and reduces plasma cholesterol levels. However, the broad substrate specificity of OST $\alpha / \beta$ raises the concern that dietary constituents, dietary supplements, or drugs could act as OST $\alpha / \beta$ inhibitors to slow bile acid export, activate FXR to induce intestinal FGF15 expression, and raise plasma cholesterol levels[20]. Because these reports are significant with regard to the mechanism of action of bile acid sequestrants, we investigated the effect against NAFLD of IBATi alone.

Alterations in the gut microbiota have been associated with an HFD in this study but little is known about the host factors that induce this alteration to the microbial population. HFD-induced BA secretion was first proposed in 2011 by Islam et al.[26] as a driving force in shaping the obesity-associated gut microbial composition. Cholic acid is the most abundant bile acid in biliary secretions in humans and HFD enhances bile acid secretion to facilitate lipid digestion. The most important secondary bile acid is deoxycholic acid, which arises from cholic acid. The conversion to secondary bile acids is mediated by a 7a-dehydroxylation reaction catalyzed by some species of Clostridium; nearly $100 \%$ of CA is converted into DCA in the large intestine. An important feature of bile acids is their strong antimicrobial activity[27]. Physiological concentrations of CA and DCA in the intestine disturb the integrity of membranes as a result of their detergent effect, leading to the leakage of ions and cellular components and, eventually, to cell death. DCA is one of the most potent antimicrobial bile acids, with ten times the bactericidal activity of CA. Thus, bile acids seem to exert a strong selective pressure on the gut microbiota and only microbial populations able to tolerate physiological concentrations of bile acids can survive in the gut. This seems especially evident when administering an HFD, causing an increased intestinal flow of bile acids[26].

There is growing concern that environmental factors, especially an HFD, have altered the genetic composition and metabolic activity of the human microbiota[28], predominantly through changes in the relative abundance of two dominant bacterial divisions, Firmicutes and Bacteroidetes. At the level of family, dramatic overgrowth of Streptococcaceae and Enterobacteriaceae has been seen in obese subjects[29]. In this study, Streptococcaceae were also increased in the gut microbiota of HFD mice and the proportion of these species decreased to the same level as normal mice after FMT from the IBATi group of mice. Due to the critical role of the gut microbiota in NAFLD development, the promotion of changes in microbiotal composition to resemble a healthier profile may be desirable in clinical practice, so we should focus on the modulatory capacity against dysbiosis and gut-liver axis activation. The administration of live bacteria with potential health benefits for the host, when provided in adequate amounts, namely probiotics, is a promising strategy to manage obesity-associated disorders. Bacteria belonging to the Lactobacillus, Streptococcus, and Bifidobacterium genera are the probiotics used most 
frequently, because of the growing body of literature supporting their health-promoting effects and because all of these genera integrate the normal human gut microenvironment[30]. In this study, IBATi promoted changes in the gut microbiota and impeded hepatic lipid accumulation. However, the protective effect of probiotics in NAFLD is not yet fully understood, although it has been proposed that the production of antibacterial substances, enhancement of epithelial barrier function, and regulation of the immune system and subsequent intestinal inflammation may be implicated[9].

\section{Conclusions}

NAFLD is highly prevalent in all counties, but therapeutic agents approved for the treatment of NAFLD are lacking. When HFD mice were treated with IBATi, their body weight gain and serum LDL levels were significantly suppressed and NAS was significantly decreased. The decrease of a-diversity in gut microbiota induced by HFD was recovered by the IBATi. IBATi ameliorated the increase of ileal Fgf15 mRNA and the decrease hepatic Cyp7a 1 mRNA in HFD mice. From FMT, the gut microbiota from HFD + IBATi mice prevented hepatic steatosis caused by HFD. From these results, IBATi improves hepatic steatosis by changing the gut microbiota dysbiosis in NAFLD model mice.

\section{Abbreviations}

NAFLD, non-alcoholic fatty liver disease; IBATi, ileal bile acid transporter inhibitor; NAS, NAFLD activity score; FMT, fecal microbiota transplantation; ASBT, apical sodium-dependent bile acid transporter; BA, bile acids; PCoA, principal coordinate analysis; HE, hematoxylin-eosin; AST, aspartate amino-transferase; ALT, alanine amino-transferase; TBA, Total Bile Acid; Tcho, total cholesterol; LDL, low-density lipoprotein; TG, triacyl glyceride; CYP7A1, cholesterol 7-a-monoxygenase; CYP8a1, cholesterol 8-a-monoxygenase; FXR, farnesoid X receptor; FGF15, fibroblast growth factor 15; HFD, high fat diet; organic solute transporter, OST.

\section{Declarations}

Ethics approval and consent to participate

All animal experiments were carried out in compliance with ICLAS guideline and Japanese regulations. The local institutional animal ethics board of Osaka Medical college approved all mouse experiments (approval number: 2019-138).

Consent for publication

Not applicable

Availability of data and material

The datasets used and/or analyzed during the current study are available, https://gofile.io/?c=Xod2Qq.

Competing interests

$\mathrm{KH}$ is consulting for EA Pharma Co., Ltd. 
Funding

This research was supported for Private University Research Branding Project in Japan \# 18.

Authors' contributions

MM and AA performed experiments, analyzed data and wrote the paper. SF, TN participated in the design of the study. TU and $\mathrm{KH}$ wrote the paper and supervised. All authors read and approved the final manuscript.

Acknowledgements

We thank Shoichi Sakaguchi at department of Microbiology and infection control in Osaka Medical College for helpful comments on this manuscripts.

\section{References}

1. Younossi Z, Anstee QM, Marietti M, Hardy T, Henry L, Eslam M, et al. Global burden of NAFLD and NASH: trends, predictions, risk factors and prevention. Nat Rev Gastroenterol Hepatol. 2018;15:1120.

2. Araujo AR, Rosso N, Bedogni G, Tiribelli C, Bellentani S. Global epidemiology of non-alcoholic fatty liver disease/non-alcoholic steatohepatitis: What we need in the future. Liver Int. 2018;38(Suppl 1):47-51.

3. Estes C, Razavi H, Loomba R, Younossi Z, Sanyal AJ. Modeling the epidemic of nonalcoholic fatty liver disease demonstrates an exponential increase in burden of disease. Hepatology. 2018;67:12333.

4. Honda Y, Kessoku T, Ogawa Y, Tomeno W, Imajo K, Fujita K, et al. Pemafibrate, a novel selective peroxisome proliferator-activated receptor alpha modulator, improves the pathogenesis in a rodent model of nonalcoholic steatohepatitis. Sci Rep. 2017;7:42477.

5. Chedid V, Vijayvargiya P, Camilleri M. Elobixibat for the treatment of constipation. Expert Rev Gastroenterol Hepatol. 2018;12:951-60.

6. Rao A, Kosters A, Mells JE, Zhang W, Setchell KD, Amanso AM, et al. Inhibition of ileal bile acid uptake protects against nonalcoholic fatty liver disease in high-fat diet-fed mice. Sci Transl Med. 2016;8:357ra122.

7. Hu H, Lin A, Kong M, Yao X, Yin M, Xia H, et al. Intestinal microbiome and NAFLD: molecular insights and therapeutic perspectives. J Gastroenterol. 2019. doi:

8. Zhou Y, Zheng T, Chen H, Li Y, Huang H, Chen W, et al. Microbial Intervention as a Novel Target in Treatment of Non-Alcoholic Fatty Liver Disease Progression. Cell Physiol Biochem. 2018;51:212335.

9. Porras D, Nistal E, Martinez-Florez S, Gonzalez-Gallego J, Garcia-Mediavilla MV, Sanchez-Campos S. Intestinal Microbiota Modulation in Obesity-Related Non-alcoholic Fatty Liver Disease. Front Physiol. 2018;9:1813. 
10. Zheng $X$, Huang F, Zhao A, Lei S, Zhang $Y$, Xie G, et al. Bile acid is a significant host factor shaping the gut microbiome of diet-induced obese mice. BMC Biol. 2017;15:120.

11. Ushiroda C, Naito Y, Takagi T, Uchiyama K, Mizushima K, Higashimura Y, et al. Green tea polyphenol (epigallocatechin-3-gallate) improves gut dysbiosis and serum bile acids dysregulation in high-fat diet-fed mice. J Clin Biochem Nutr. 2019;65:34-46.

12. Simren M, Bajor A, Gillberg PG, Rudling M, Abrahamsson H. Randomised clinical trial: The ileal bile acid transporter inhibitor $A 3309$ vs. placebo in patients with chronic idiopathic constipation-a double-blind study. Aliment Pharmacol Ther. 2011;34:41-50.

13. Acosta A, Camilleri M. Elobixibat and its potential role in chronic idiopathic constipation. Therap Adv Gastroenterol. 2014;7:167-75.

14. Routy B, Le Chatelier E, Derosa L, Duong CPM, Alou MT, Daillere R, et al. Gut microbiome influences efficacy of PD-1-based immunotherapy against epithelial tumors. Science. 2018;359:91-7.

15. Inoue R, Sawai T, Sawai C, Nakatani M, Romero-Perez GA, Ozeki M, et al. A preliminary study of gut dysbiosis in children with food allergy. Biosci Biotechnol Biochem. 2017;81:2396-9.

16. Kleiner DE, Brunt EM, Van Natta M, Behling C, Contos MJ, Cummings OW, et al. Design and validation of a histological scoring system for nonalcoholic fatty liver disease. Hepatology. 2005;41:1313-21.

17. Brunt EM, Kleiner DE, Wilson LA, Belt P, Neuschwander-Tetri BA. Nonalcoholic fatty liver disease (NAFLD) activity score and the histopathologic diagnosis in NAFLD: distinct clinicopathologic meanings. Hepatology. 2011;53:810-20.

18. Schumacher JD, Kong B, Pan Y, Zhan L, Sun R, Aa J, et al. The effect of fibroblast growth factor 15 deficiency on the development of high fat diet induced non-alcoholic steatohepatitis. Toxicol Appl Pharmacol. 2017;330:1-8.

19. Kramer W, Girbig F, Glombik H, Corsiero D, Stengelin S, Weyland C. Identification of a ligand-binding site in the $\mathrm{Na}+$ /bile acid cotransporting protein from rabbit ileum. J Biol Chem. 2001;276:36020-7.

20. Dawson PA, Haywood J, Craddock AL, Wilson M, Tietjen M, Kluckman K, et al. Targeted deletion of the ileal bile acid transporter eliminates enterohepatic cycling of bile acids in mice. J Biol Chem. 2003;278:33920-7.

21. Out C, Patankar JV, Doktorova M, Boesjes M, Bos T, de Boer S, et al. Gut microbiota inhibit Asbtdependent intestinal bile acid reabsorption via Gata4. J Hepatol. 2015;63:697-704.

22. Annaba F, Sarwar Z, Gill RK, Ghosh A, Saksena S, Borthakur A, et al. Enteropathogenic Escherichia coli inhibits ileal sodium-dependent bile acid transporter ASBT. Am J Physiol Gastrointest Liver Physiol. 2012;302:G1216-22.

23. Rao A, Haywood J, Craddock AL, Belinsky MG, Kruh GD, Dawson PA. The organic solute transporter alpha-beta, Ostalpha-Ostbeta, is essential for intestinal bile acid transport and homeostasis. Proc Natl Acad Sci U S A. 2008;105:3891-6.

24. Ballatori N, Fang F, Christian WV, Li N, Hammond CL. Ostalpha-Ostbeta is required for bile acid and conjugated steroid disposition in the intestine, kidney, and liver. Am J Physiol Gastrointest Liver Physiol. 2008;295:G179-g86. 
25. Lan T, Rao A, Haywood J, Kock ND, Dawson PA. Mouse organic solute transporter alpha deficiency alters FGF15 expression and bile acid metabolism. J Hepatol. 2012;57:359-65.

26. Islam KB, Fukiya S, Hagio M, Fujii N, Ishizuka S, Ooka T, et al. Bile acid is a host factor that regulates the composition of the cecal microbiota in rats. Gastroenterology. 2011;141:1773-81.

27. Kurdi P, Kawanishi K, Mizutani K, Yokota A. Mechanism of growth inhibition by free bile acids in lactobacilli and bifidobacteria. J Bacteriol. 2006;188:1979-86.

28. David LA, Maurice CF, Carmody RN, Gootenberg DB, Button JE, Wolfe BE, et al. Diet rapidly and reproducibly alters the human gut microbiome. Nature. 2014;505:559-63.

29. Shen J, Obin MS, Zhao L. The gut microbiota, obesity and insulin resistance. Mol Aspects Med. 2013;34:39-58.

30. Iacono A, Raso GM, Canani RB, Calignano A, Meli R. Probiotics as an emerging therapeutic strategy to treat NAFLD: focus on molecular and biochemical mechanisms. J Nutr Biochem. 2011;22:699711.

Figures 
Fig. 1
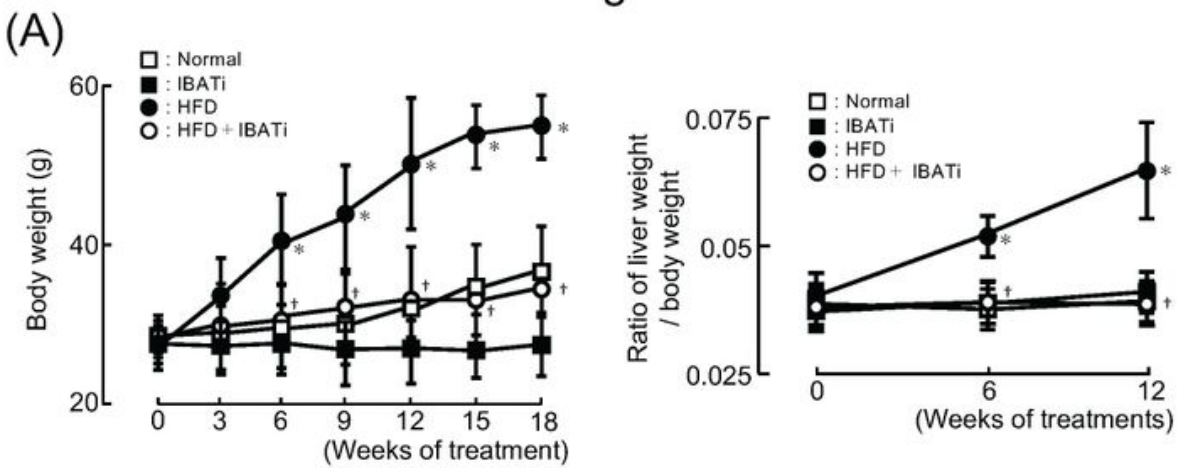

(B)
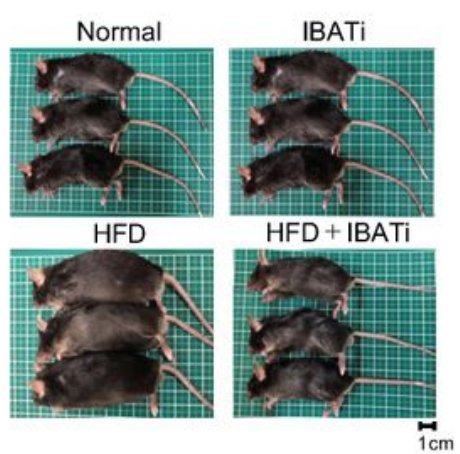

(C)

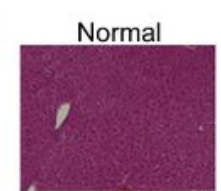

IBATi
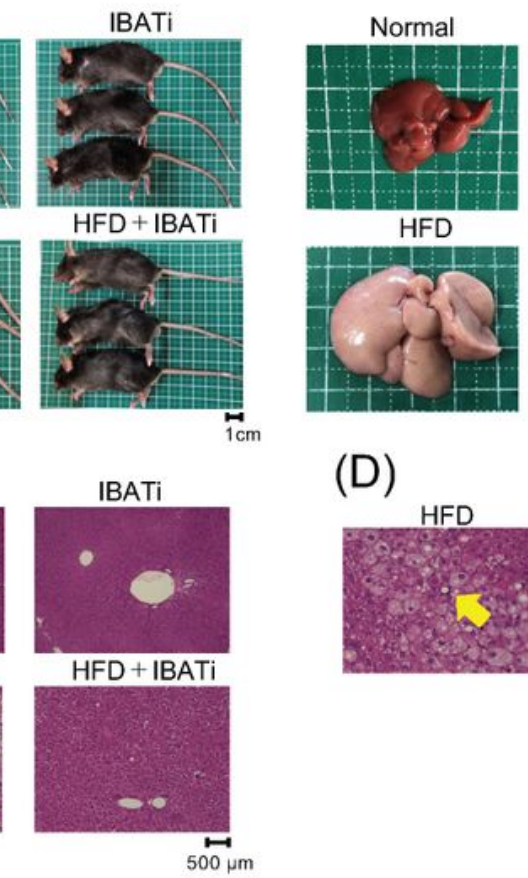

(D)
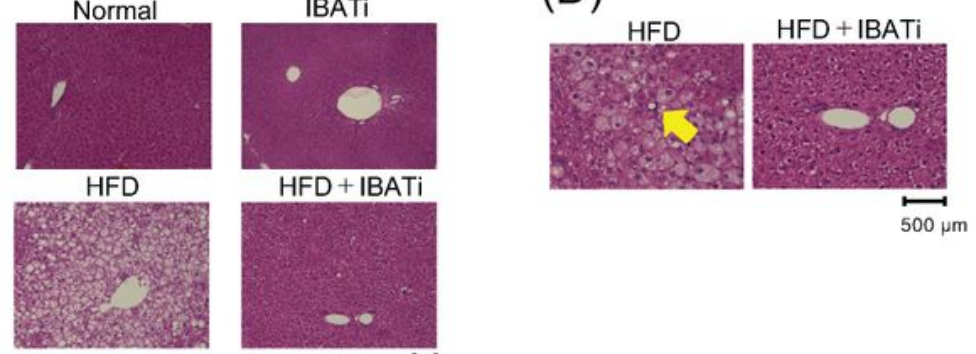

$(\mathrm{E})$

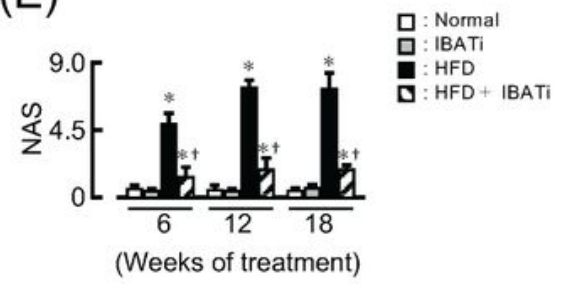

(F)

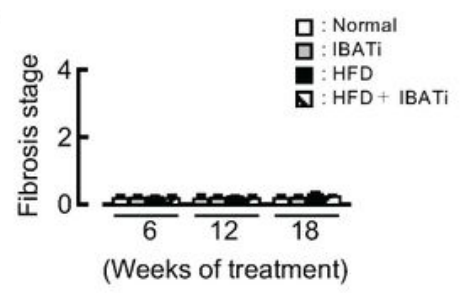

\section{Figure 1}

Inhibitory effect of IBATi on the HFD-induced NAFLD (A) Time course of mouse body weights. The HFD + IBATi group of mice was fed an HFD and treated with IBATi $(2.5 \square \mathrm{mol} / \mathrm{kg})($ Open circles: $\mathrm{n}=6)$. The HFD group of mice was fed an HFD without any treatment (filled circles: $n=6$ ). The IBATi group of mice was fed a normal diet and treated with IBATi $(2.5 \square \mathrm{mol} / \mathrm{kg}$ ) (filled squares: $\mathrm{n}=6)$. The normal group of mice was fed a normal diet without any treatment (open squares: $n=6$ ). (B) Macroscopic photographs from 
each group of mice. (C) Time course of mouse liver weight / body weight ratios. (D) Representative macroscopic photographs of livers from 18 week-old mice. (E) Hematoxylin \& eosin (HE)-stained liver sections. (F) The hepatic crown structure in the liver was detected only in liver sections from the HFD group. Photographs are of HE staining of liver sections from representatives of each group (scale bar = $500 \rrbracket m$ ). (G) NAFLD activity scores (NAS) of liver sections were evaluated. (H) Fibrosis stages were evaluated. Significant differences from the normal group are denoted $*(p<0.05)$ and the those with the HFD group are denoted $t(p<0.05)$.

Fig. 2

(A)
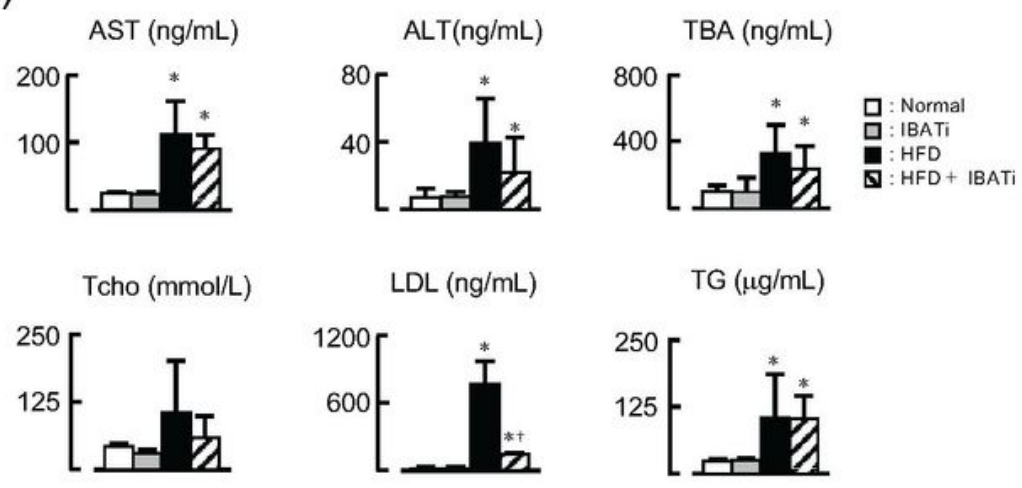

(B)
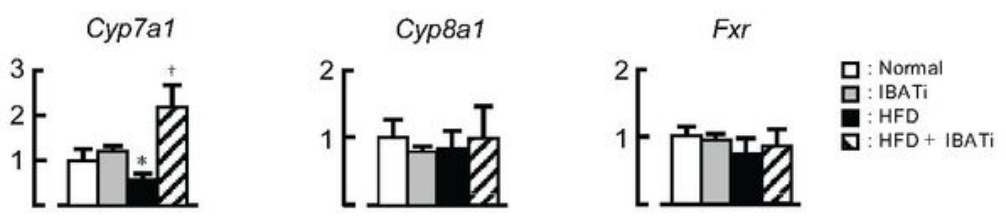

(C)
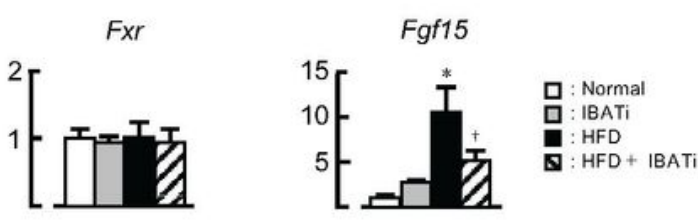
Figure 2

Biochemical parameters of the mice (A) Biochemical parameters of serum from each group of 18 weekold mice were assayed using ELISA. (B) Hepatic mRNA and (C) ileal mRNA expression from each group of 18 week-old mice were measured by RT-PCR. Significant differences from the normal group are denoted $*(p<0.05)$ and the those with the HFD group are denoted $+(p<0.05)$.

Fig. 3

\section{(A)}

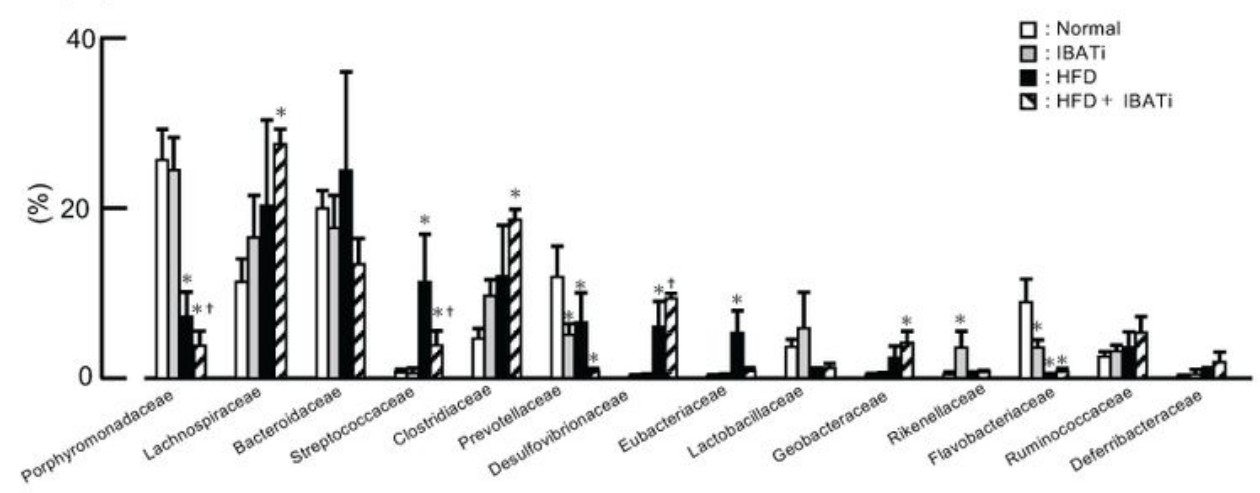

(B)

(C)

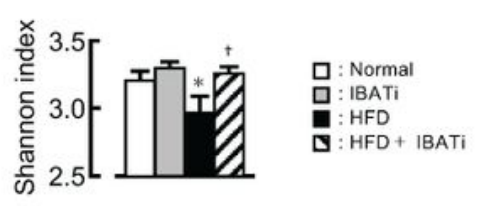

(D)
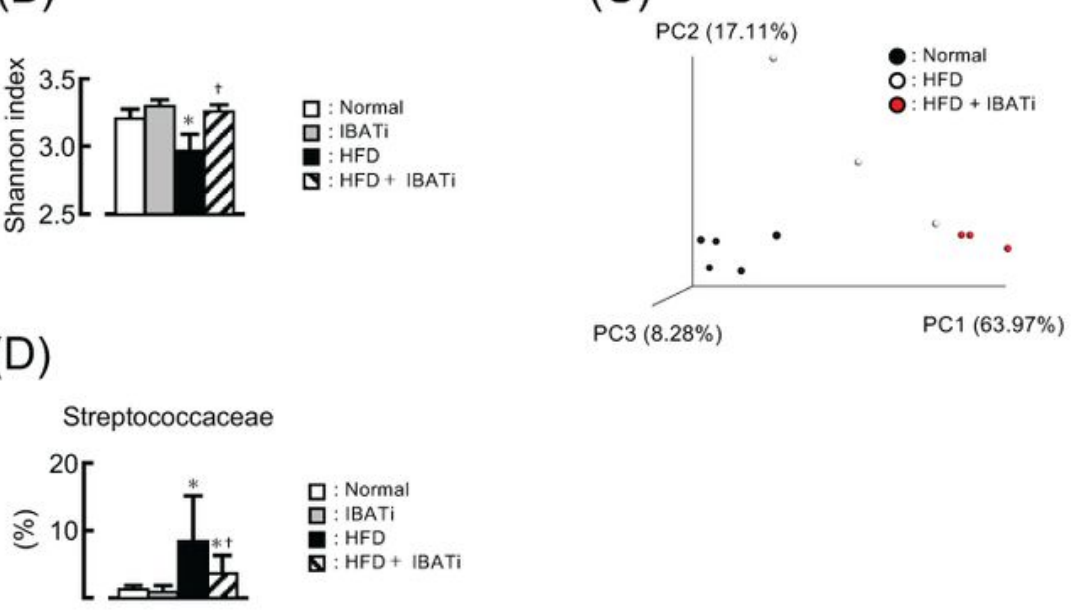
Effects of IBATi administration on gut microbiota The microbial compositions of the feces were analyzed using lon reporter-based 16S rRNA sequencing. Values are expressed as the means and SEM of three or four mice in each group. (A) The microbial composition at the family level. (B) The Shannon index (OUT evenness estimation). (C) PCoA plot of unweighted Bray Curtis data. The overall structures of the

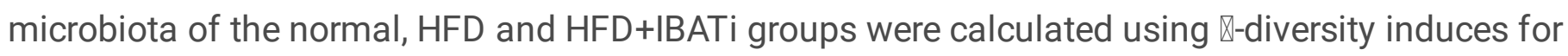
unweighted Bray Curtis distances. (D) Percentages of Streptococcaceae. Significant differences compared to the normal group are denoted $*(p<0.05)$, and the those with the HFD group are denoted $+(p$ $<0.05)$. 
Fig. 4
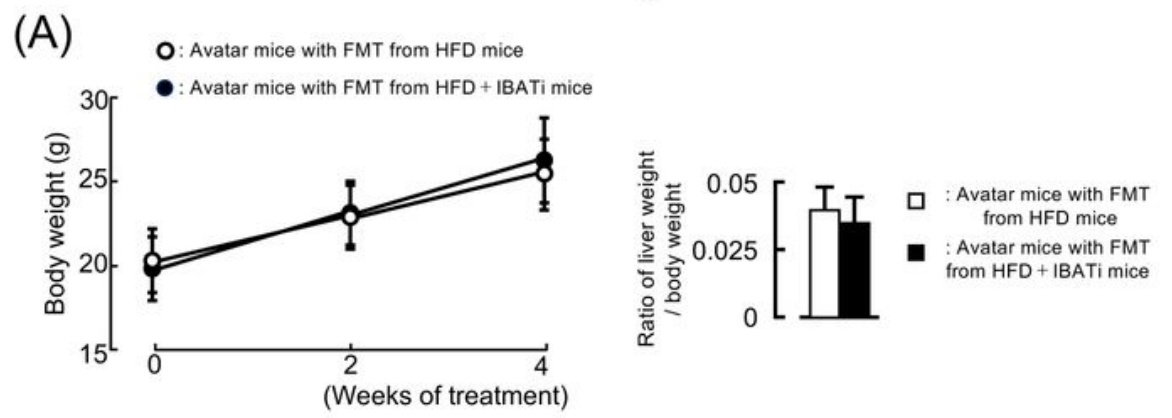

(B)
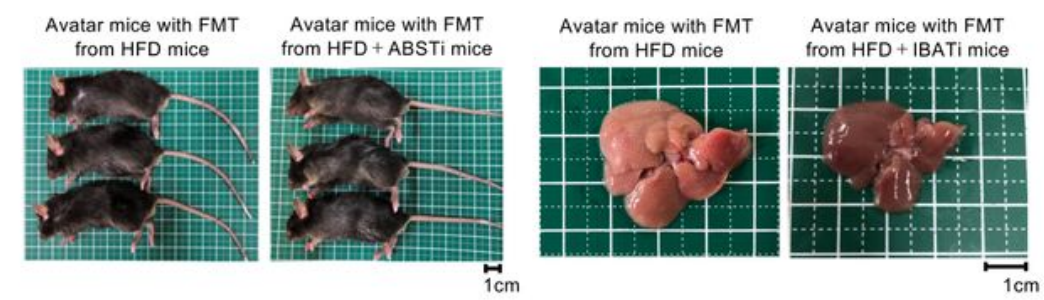

(C)

(D)
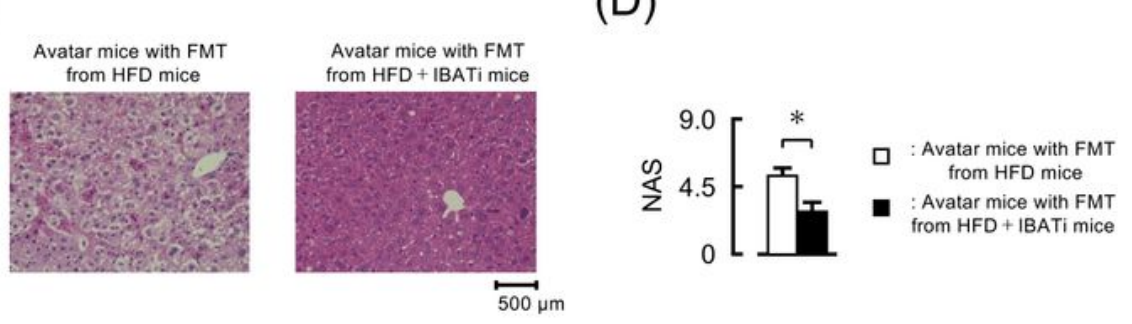

(E)

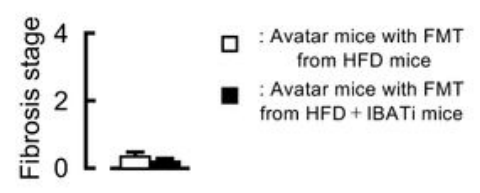

\section{Figure 4}

Fecal microbiota transplantation (FMT) of stool samples from HFD-IBATi mice protected decontaminated mice with an HFD diet from hepatic steatosis. FMT was performed in avatar mice 48 hours after ATB. Six week-old avatar mice were fed an HFD diet for 8 weeks. These avatar mice were treated by ATB at 9 weeks of age. FMT was performed in avatar mice 48 hours after ATB. These mice were prepared by oral gavage of feces harvested from the HFD or HFD+IBATi groups of 18 week-old mice every two days for a 
week. After FMT, both groups of avatar mice were fed an HFD diet for 4 weeks (avatar mice with FMT from HFD groups of mice, open circles: $n=6$; avatar mice with HFD+IBATi mice, closed circles: $n=6)(A)$ Time course of mouse body weights. (B) Macroscopic photographs from each group of avatar mice. (C) Liver weight / body weight ratios of both groups. (D) Representative macroscopic photographs of the livers. (E) Hematoxylin \& eosin (HE)-stained liver sections. Photographs are of HE staining of liver sections from representatives of each group (scale bar $=500 \square \mathrm{m}$ ). $(\mathrm{F})$ NAS of liver sections were evaluated. (G) Fibrosis stages were evaluated. Significant differences compared between groups are denoted $*(p<0.05)$. 
Fig. 5

(A)
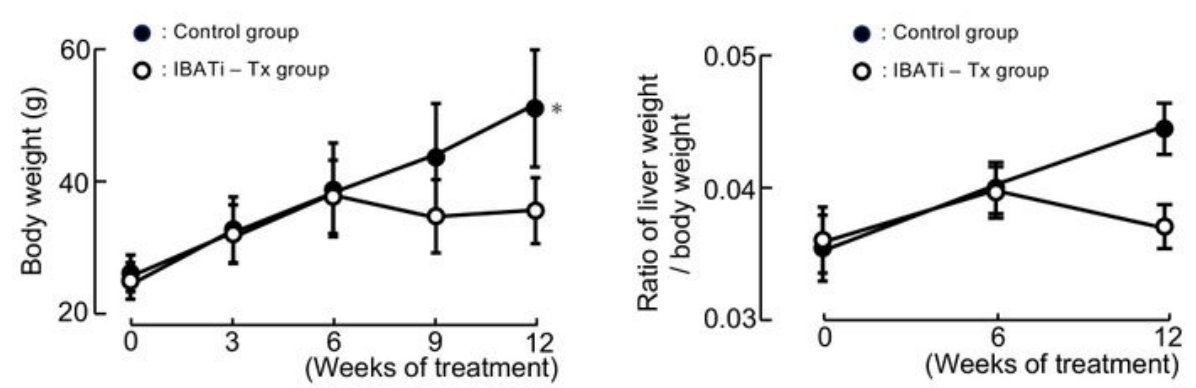

(B)
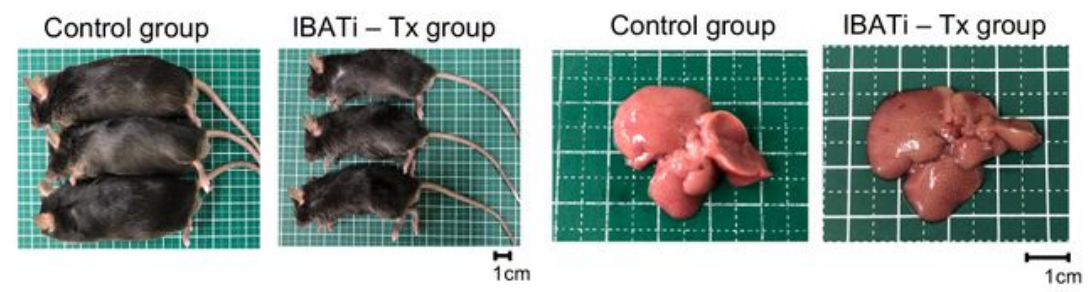

(C)

(D)
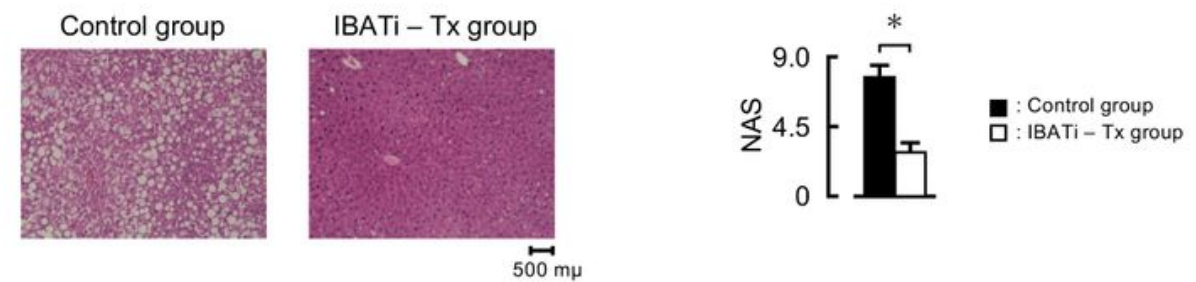

(E)

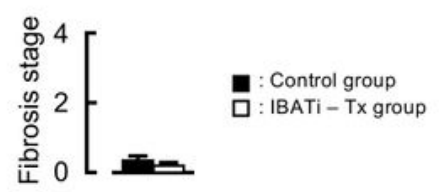

\section{Figure 5}

Therapeutic effects of IBATi on HFD induced NAFLD mice. C57/BL mice were fed an HFD diet for 12 weeks. To examine the therapeutic effects of IBATi, these mice were treated orally with IBATi from 6 to 12 weeks (IBATi-Tx group). For the control group, equivalent mice were subjected orally to the same dose of PBS. Time course of mouse body weights (A) and representative macroscopic photographs from each group of mice (B), liver weight / body weight ratios (C) and representative macroscopic photographs of 
the livers (D) (IBATi-Tx group, open circles: $n=6$; control group, closed circles: $n=6$ ). (E) Hematoxylin \& eosin (HE)-stained liver sections. Photographs are of HE staining of liver sections from representatives of each group (scale bar $=500 \square \mathrm{m}$ ). (F) NAFLD activity scores (NAS) of liver sections were evaluated. $(G)$ Fibrosis stages were evaluated. Significant differences compared between groups are denoted $*(p<$ 0.05).

Fig. 6

(A)

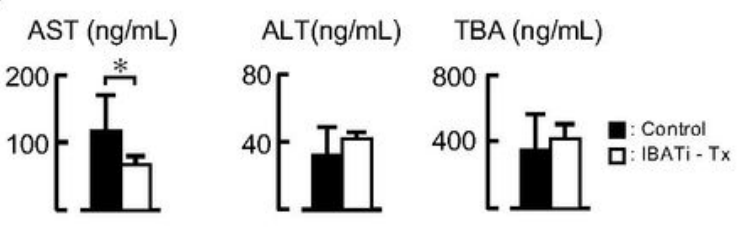

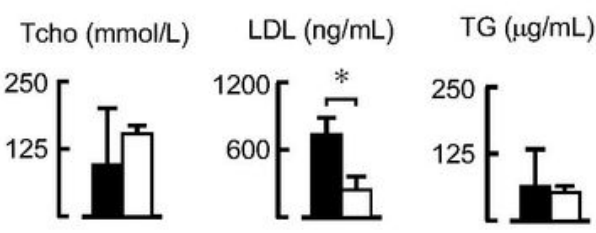

(B)

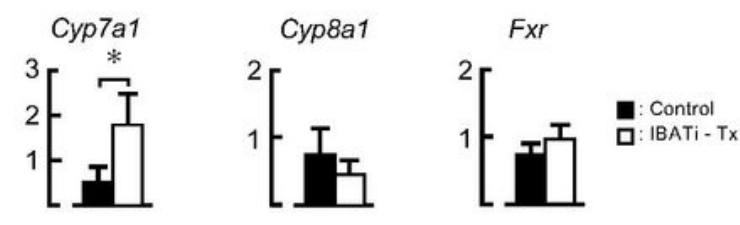

(C)

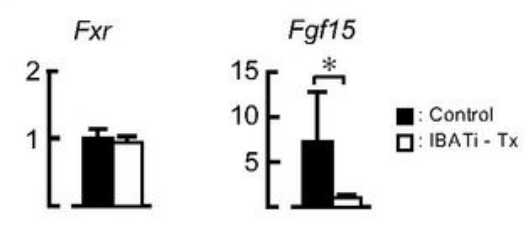

Figure 6 
Biochemical parameters of the mice of IBATi-TX mice Biochemical parameters of sera from each group of control mice and IBATi-Tx mice were assayed using ELISA. (B) Hepatic mRNA and (C) ileal mRNA expression from each group of mice were measured by rt-PCR. Significant differences compared to the control group are denoted $*(p<0.05)$.

\section{Supplementary Files}

This is a list of supplementary files associated with this preprint. Click to download.

- 20200406supplementalfigure1.pdf

- 20200406supplementalfigure1.pdf 